LA-UR-99-5985

Approved for public release; distribution is unlimited.

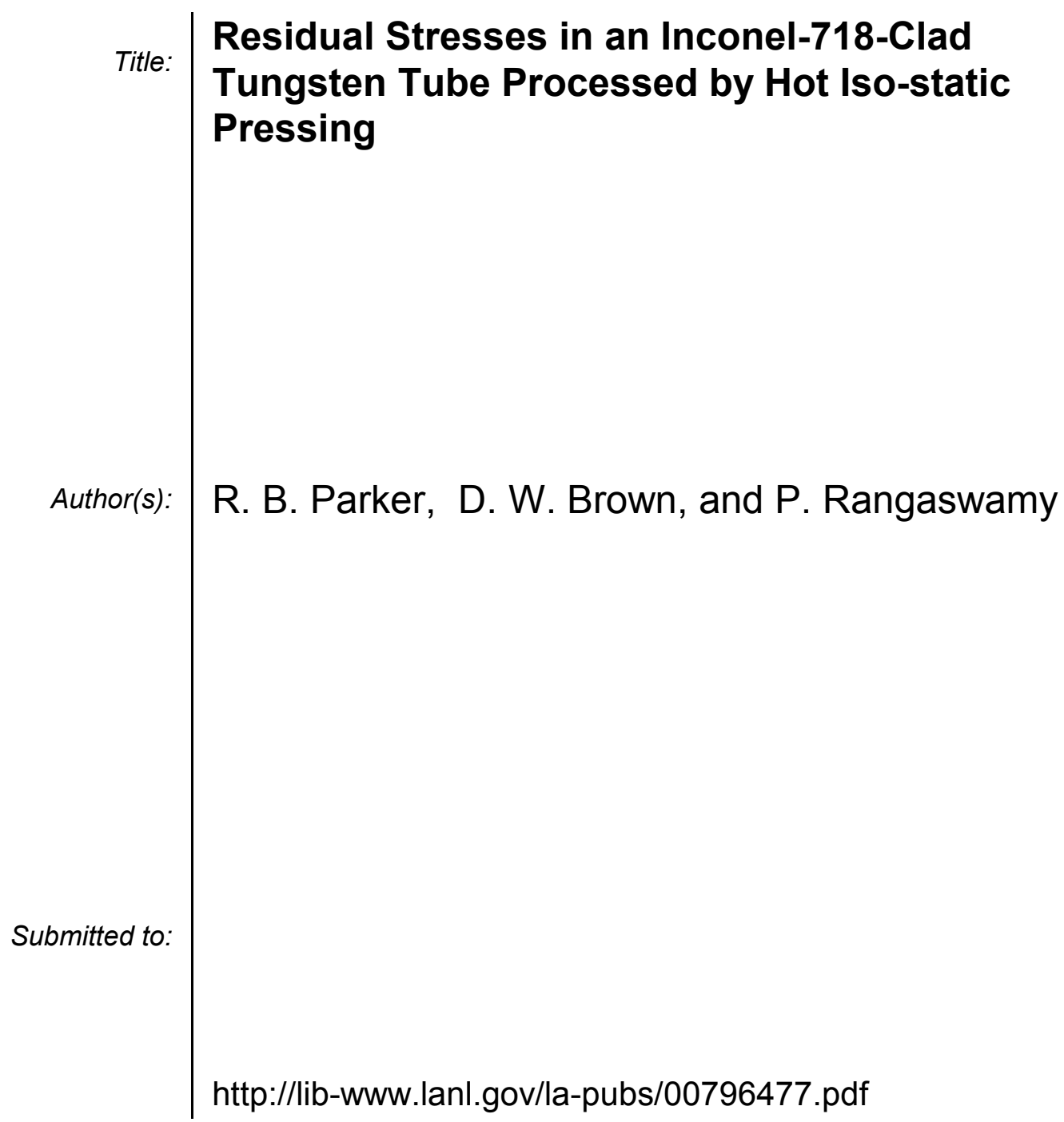

Los Alamos National Laboratory, an affirmative action/equal opportunity employer, is operated by the University of California for the U.S. Department of Energy under contract W-7405-ENG-36. By acceptance of this article, the publisher recognizes that the U.S. Government retains a nonexclusive, royaltyfree license to publish or reproduce the published form of this contribution, or to allow others to do so, for U.S. Government purposes. Los Alamos National Laboratory requests that the publisher identify this article as work performed under the auspices of the U.S. Department of Energy. Los Alamos National Laboratory strongly supports academic freedom and a researcher's right to publish; as an institution, however, the Laboratory does not endorse the viewpoint of a publication or guarantee its technical correctness. 


\title{
Residual Stresses in an Inconel-718-Clad Tungsten Tube Processed by Hot Iso-static Pressing
}

\author{
R. B. Parker ${ }^{1}$ D. W. Brown ${ }^{2}$, and P. Rangaswamy ${ }^{3}$ \\ Los Alamos National Laboratory \\ Los Alamos, NM 87545
}

Keywords: Residual Stresses, Hot Iso-static Pressing, HIP, Finite Element, FEA, X-ray diffraction, XRD.

\begin{abstract}
The Accelerator Production of Tritium (APT) facility has been developed as a possible tritium supply to the United States Nuclear Weapon Stockpile. To produce tritium, tungsten targets are bombarded with high-energy protons producing neutrons, via spallation reactions. The neutrons produced by these reactions are then captured in a ${ }^{3} \mathrm{He}$ blanket to produce tritium. Design requirements and safety consideration do not allow coolant to be in direct contact with the tungsten, necessitating cladding of the rods. Inconel 718 was chosen as the cladding material for its superior mechanical, thermal, and neutronic properties. Because the cladding must be in intimate contact with the tungsten at operating temperatures, the materials are diffusion bonded by Hot Iso-static Pressing (HIP) at $1080^{\circ} \mathrm{C}$. However, the difference in thermal expansion coefficients of inconel 718 and tungsten produce large residual stresses upon cooling. We have utilized X-ray diffraction (XRD) to study a tungsten tube clad with inconel 718 on both the inner and outer surface. The classical XRD ( $\left.d v s \cdot \sin ^{2} \psi\right)$ technique was used to determine the stresses in the outer layer of inconel 718. These results are used to benchmark Finite Element Analysis (FEA) calculations, and a direct comparison is made in this paper.
\end{abstract}

\section{Introduction}

The production of tritium within the APT facility will rely upon the preferential absorption of neutrons by ${ }^{3} \mathrm{He}$, an isotope of Helium, to produce tritium. Neutrons are produced within the target region by spallation reactions between high-energy protons and tungsten. To optimize the production of neutrons within the target, the tungsten is split apart and spaced to allow neutrons to escape the target. To produce the required geometry and cool the target, a ladder-rung arrangement is used. Within the rungs of the ladder, inconel-718-clad concentric tungsten tubes will be placed with coolant circulated around them.

The concentric tube arrangement was chosen during the design process to reduce analysis uncertainty and increase thermal-hydraulic safety margins. To ensure safe operations the tungsten target cannot be allowed to come in contact with the water coolant during off normal or accident conditions, thus necessitating cladding of the tungsten tubes. Safe operating conditions require a target geometry which maintains a geometry capable of being cooled during normal and off-normal operations. To ensure this condition, the cladding must remain in intimate contact with the tungsten at all times. However, the higher coefficient of thermal expansion (CTE) of inconel 718, coupled with the geometric layout of the tube, requires the cladding to be bonded to the tungsten. Hot isostatic pressing of the clad rods was proposed to diffusion bond the inconel 718 to the tungsten. However, the HIP process generates residual stresses within the tube that must be accouuring plant 
operations. In this research, the objective was to apply finite element modeling to simulate the evolution of thermal residual stresses during the HIP process and to benchmark these predictions with XRD measurements after the completion of the consolidation process. Once benchmarked, the finite element model can be used for subsequent analysis determining the response of the target to plant normal operational and safety-case conditions.

\section{Numerical Simulation of Residual Stresses using Finite Element Modeling (FEM)}

Physical Geometry. The clad tube studied by this analysis is manufactured by sliding a 16.027 $\mathrm{mm}$ inside diameter (ID) tungsten tube into an inconel 718 tube with a $0.152 \mathrm{~mm}$ wall thickness and a nominal $16.078 \mathrm{~mm}$ inside diameter. This creates a nominal $0.0254^{\circ} \mathrm{mm}$ on-radius clearance between the outer cladding and tungsten. This gap is explicitly modeled within the finite element models to accurately predict processing stresses. A second $11.862^{\circ} \mathrm{mm} \mathrm{ID,} 0.152 \mathrm{~mm}$ wall thickness inconel 718 tube is slipped inside the tungsten cylinder with the same nominal gap clearance. End caps are then electron-beam welded on the ends of the tube to completely encase the tungsten tube in inconel 718.

HIP Process. After assembly and welding the clad tube is placed within a hot isostatic press and

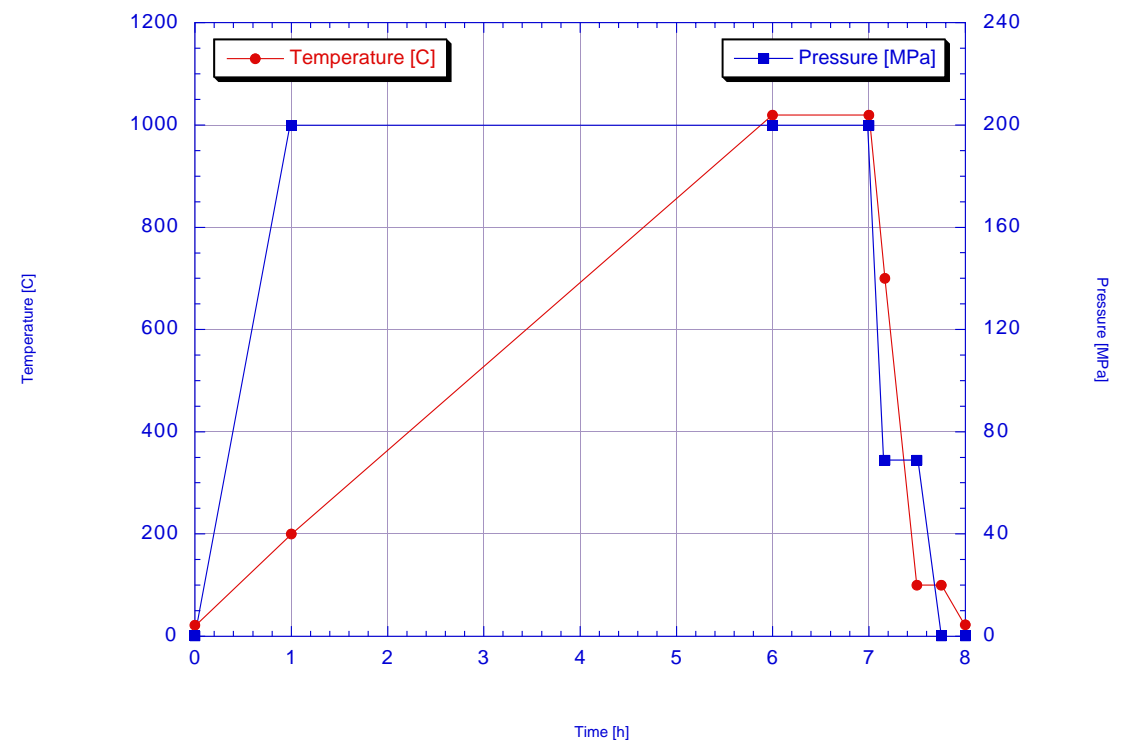

Figure 1. HIP cycle parameters showing pressure and temperature variation with time. subjected to the pressure and time history shown in Fig. 1. Externally applying an appreciable pressure to the assembly forces the inconel 718 cladding to entirely contact the tungsten. At some undetermined time during the cycle, they become bonded together.

Finite Element Model. A two dimensional, axisymmetric FEM with one-half symmetry was developed to model the HIP process. The general purpose, commercially available finite element package, ABAQUS, was chosen to numerically simulate the HIP process. It was chosen for its capability to solve thermal-mechanically coupled problems, and its ability to explicitly model contact between bodies with friction. The model consists of 12,586 4-node axisymmetric elements, and 14,955 nodes to model the rod.

FEM Constitutive Model. The tungsten target material has a minimum purity of $99.9 \%$, and was obtained from a commercial vendor. A linear elastic constitutive model was used for the tungsten, as shown in Table 1. High-temperature data was unavailable for the tungsten, so it was assumed that these properties prevailed at all temperatures during the analysis. It should be noted that room temperature properties suggest the tungsten behaves as a brittle material, exhibiting no plasticity [1]. 
Table 1. Thermal and mechanical Properties of Pure Tungsten.

\begin{tabular}{|c|c|c|c|c|}
\hline Density & Youngs Modulus & Yield Strength & Ultimate Strength & CTE \\
\hline $19.7\left[\mathrm{~g} / \mathrm{cm}^{3}\right]$ & $404[\mathrm{GPa}]$ & $1507[\mathrm{GPa}]$ & $1507[\mathrm{GPa}]$ & $4.5 \times 10^{-6}[/ \mathrm{K}]$ \\
\hline
\end{tabular}

Annealed inconel 718 was chosen for the cladding. This material was chosen for its superior thermal and neutronic properties. Inconel 718 is a nickel-based super alloy. The constitutive model for the inconel 718 cladding consists of explicitly defined elastic-plastic true stress versus strain curves at various temperatures. The engineering stress versus strain curves used to develop the constitutive relationship are shown in Fig. 2 [2], along with the temperature-dependent elastic modulus, Poisson s ratio, and CTE [3]. Note that ABAQUS by default, uses a Von Mises yield
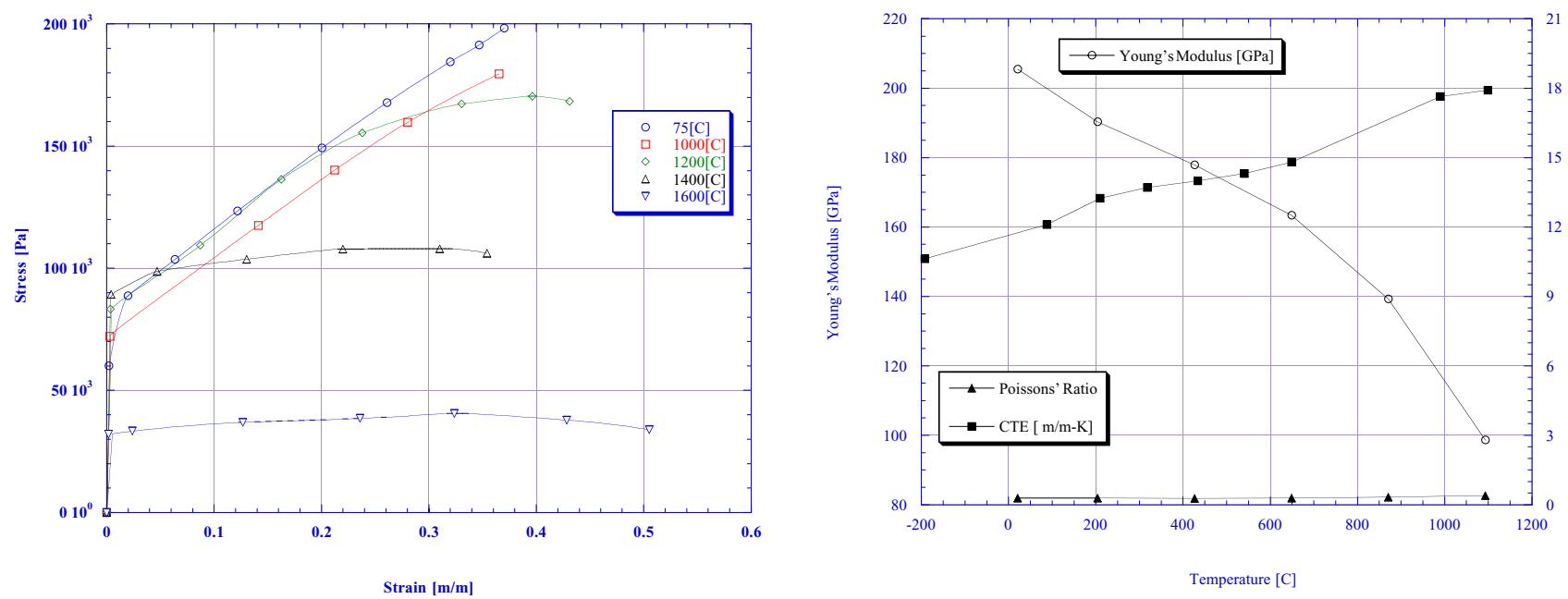

Figure 2. Engineering properties for inconel 718 at various temperatures.

surface and was used for all calculations. ABAQUS also requires isotropic hardening flow rule for coupled temperature - displacement analysis. Although isotropic hardening may not produce as accurate results as kinematic hardening for cyclic plastic loading, the coupled solution technique was chosen for its benefits and versatility, thus limiting the plasticity model to isotropic hardening. ABAQUS also determines parameters by linearly interpolating to temperatures between curves, and assumes perfect plasticity beyond the last defined point on any curve for this material model.

The ABAQUS power law model [4] was used to simulate the high-temperature creep response of the inconel clad. This process was included in the analysis to describe the stress relief effects material creep provides during the HIP cycle. The form of this material model is shown below:

$$
\dot{\bar{\varepsilon}}=A \sigma^{n} t^{m} \text {. }
$$

where:

$\dot{\bar{\varepsilon}}$ is the creep strain rate $[1 / \mathrm{s}]$,

$\sigma$ is the uniaxial equivalent deviatoric stress $[\mathrm{Pa}]$,

$\mathrm{t}$ is the total time [s],

$\mathrm{A}, \mathrm{m}, \mathrm{n}$ are user defined material dependent properties.

The user-defined coefficients for inconel 718 were determined by a linear regression fit of available data, [5] and are listed in Table 2. 
Table 2. ABAQUS User Defined Power Law Creep Parameters for Inconel 718

\begin{tabular}{|c|c|c|c|}
\hline $\begin{array}{c}\text { Temperature } \\
{[\mathrm{C}]}\end{array}$ & $\mathrm{A}$ & $\mathrm{n}$ & $\mathrm{m}$ \\
\hline \hline 24 & $8.3920 \times 10^{-93}$ & 17.170 & -0.9342 \\
\hline 538 & $1.645 \times 10^{-12}$ & 12.922 & -0.3859 \\
\hline 649 & $4.5579 \times 10^{-59}$ & 10.587 & -0.3859 \\
\hline 760 & $1.9145 \times 10^{-51}$ & 5.4141 & -0.3054 \\
\hline
\end{tabular}

FEM Analysis Procedures. The HIP process cycle, shown in Fig. 1, was divided into numerical steps for the finite element analysis. All of the steps were coupled temperature-displacement, (carried to steady-state) implicitly integrated steps, except for the high-temperature explicit viscoplasticity (creep) step. During heat up, contact between the tungsten and inconel 718 was maintained by applying pressure, and modeled using a sliding interface with a friction coefficient of 0.95. [6] The 0.95 sliding friction was maintained through the visco-plasticity step. After the viscoplastic step the friction was then changed to a tied contact condition, prior to cooling the tube back to room temperature. The ABAQUS Coulomb friction model was used throughout the process. We used these analysis steps to emulate the diffusion bond between inconel 718 and tungsten. This approach allows for a direct comparison of the calculated final residual stress state with XRD residual stress measurements upon the completion of the HIP cycle. It also allows some flexibility to numerically simulate different interface and HIP process conditions. These simulations would provide invaluable information and design guidance to the preliminary design of the APT target.

\section{Evaluation of Residual Stresses using X-ray Diffraction}

X-ray diffraction. The measurement of residual stress by X-ray diffraction utilizes the spacing of the lattice planes as a gauge length for measuring strain [7,8]. A change in stress results in a modification of the interplanar spacing, which alters the angular position of the diffraction peaks. The interplanar spacing of a specific set of planes is obtained from grains of different orientations to the surface normal. The variation of the interatomic spacing is determined as a function of $\psi$, the angle between the surface normal and the direction of the measured strain. This is determined by tilting the sample (by an angle of $\psi$ ) and rotating the specimen (by an angle of $\phi$ ) with respect to the incident X-ray beam. The change in interplanar spacing as a function of orientation $(\phi, \psi)$ is due to surface strains which can be related to the surface stresses [8]. This is also known as the classical $d_{\phi \psi}$ versus $\sin ^{2} \psi$ method of measuring near surface stresses using X-ray diffraction. Accordingly, for known values of $d_{\phi \psi}$ and $d_{0}$ (strain-free), the average strain along the $\phi-\psi$ direction with respect to the surface normal $\left(\varepsilon_{33}\right)$ can be written as [7]

$$
\left(\varepsilon_{33}\right)_{\phi, \psi}=\frac{d_{\phi, \psi}-d_{0}}{d_{0}},
$$

where $(\varepsilon)_{\phi, \psi}$ can be related to the surface stresses by the conventional elasticity equations. For classical X-ray stress analysis, these strains are a linear function of the angle $\psi$, and the surface stress state in the sample can be determined by a linear relationship between $\left(\varepsilon_{33}\right)_{\phi, \psi}$ and $\sin ^{2} \psi[7]$.

$$
\left(\varepsilon_{33}\right)_{\phi, \psi}=\frac{\mathrm{a}_{\phi, \psi}-\mathrm{a}_{0}}{\mathrm{~d}_{0}}=\frac{(1+v)}{\mathrm{E}} \sigma_{\phi} \sin ^{2} \psi+{ }_{\mathrm{E}}^{\mathrm{v}}\left(\sigma_{11}+\sigma_{22}\right)
$$


where $v$ (Poisson s ratio) and E (Young s modulus) are elastic constants and $\sigma_{11} \cos ^{2} \phi+\sigma_{11} \sin ^{2} \phi=$ $\sigma_{\phi}$ and the stresses are in the direction of $\sigma_{\phi}$.

If $\left(\varepsilon_{33}\right)$ is plotted versus $\sin ^{2} \psi$, then equation (3) represents the equation of a straight line with the slope proportional to $\sigma_{\phi}$. In this study, the assumption of a biaxial plane stress state is generally valid since the average depth of penetration of $\mathrm{Cu}-\mathrm{K}_{\alpha} \mathrm{X}$-rays in this alloy has been computed to be between approximately 11 microns (at $\psi=0^{\circ}$ ) and 6 microns (at $\psi=60^{\circ}$ ).

The stress $\sigma_{\phi}$ measured by the X-ray method contains both the macrostress and microstress component. The raw data consists of plots of $d$ versus $\sin ^{2} \psi$ which were fairly linear also known as regular. The slopes of the linear plots were used to determine the surface resid ual stress along the specific orientation of measurement. For a regular or linear behavior of $d v s$. $\sin ^{2} \psi$ the stress $\sigma_{\phi}$ represent a uniform stress distribution in the measured area of the sample. This also implies that the microstress components cancel out due to equilibrium conditions within the sampling volume. Therefore, the remaining stress component is the macrostress. This macro-stress component can be used for validating the predictions from analytical or finite element models.

Measurement Procedure Residual stresses were measured in the inconel 718 along the axial (parallel to the axis of the tube) and hoop (tangent to the circumference of the tube) direction for comparison with predictions from FEM models. These measurements were made using a Huber stress goniometer with X-rays generated from an $18 \mathrm{~kW}$ Scintag rotating anode located at the Lujan Center (LANSCE-12) at Los Alamos National Laboratory.

All measurements used $\mathrm{Cu}-\mathrm{K}_{\alpha}$ radiation (wavelength 1.54 ). Initial measurements involved recording $2 \theta$ scans from $40^{\circ}$ to $160^{\circ}$ for characterization of the material and identifying the diffraction peaks in the high angle $\left(2 \theta>135^{\circ}\right)$. The diffraction pattern revealed a single-phase material having a face centered cubic structure (fm $3 \mathrm{~m})$. Two diffraction planes (331) and (420) at $2 \theta$ approximately $137^{\circ}$ and $145^{\circ}$ respectively, were identified for residual stress measurements. The $\mathrm{X}$-ray beam was collimated using a $2 \mathrm{~mm}$ diameter collimator on the incident side to the sample for axial stress measurements. The distance from the sample surface to the tip of the collimator was 40 $\mathrm{mm}$. After passing through receiving slits the scattered beam was monochromated by the (200) plane of a graphite single crystal and detected by a scintillation detector.

Measurements of the hoop and axial stress were performed along the length of a Inconel 718 clad tungsten tube of size $56 \mathrm{~mm}$ long x $16 \mathrm{~mm}$ in diameter in this study. For the purposes of this study, the center of the rod will be labelled $x=0$, with the ends being at $\pm 28 \mathrm{~mm}$. Stresses were calculated from the slopes of the $\mathrm{d} v \sin ^{2} \psi$ plots using an X-ray elastic constant of $3.5 \times 10^{-6} \mathrm{MPa}$ [8].

\section{Results}

Figure 3 shows plots of the predicted and measured axial and hoop residual stresses as a function of position along the outer surface of the clad tube. While the magnitude of the residual stresses do not agree, the functional form of the predicted and measured stress agree nicely. Proximal to the end cap and towards the end of the clad tube both the predicted and measured residual stresses are compressive. Roughly $5 \mathrm{~mm}$ from either end the residual stresses change sense and become tensile. Within $\pm 20 \mathrm{~mm}$ of the center of the tube the measured residual stresses are uniform and equal to 190 and $160 \mathrm{MPa}$ in the axial and hoop direction respectively. The FEM also predicts uniform residual stresses over this range of positions, but with a magnitude of 505 and $495 \mathrm{MPa}$ in the axial and hoop directions respectively. 


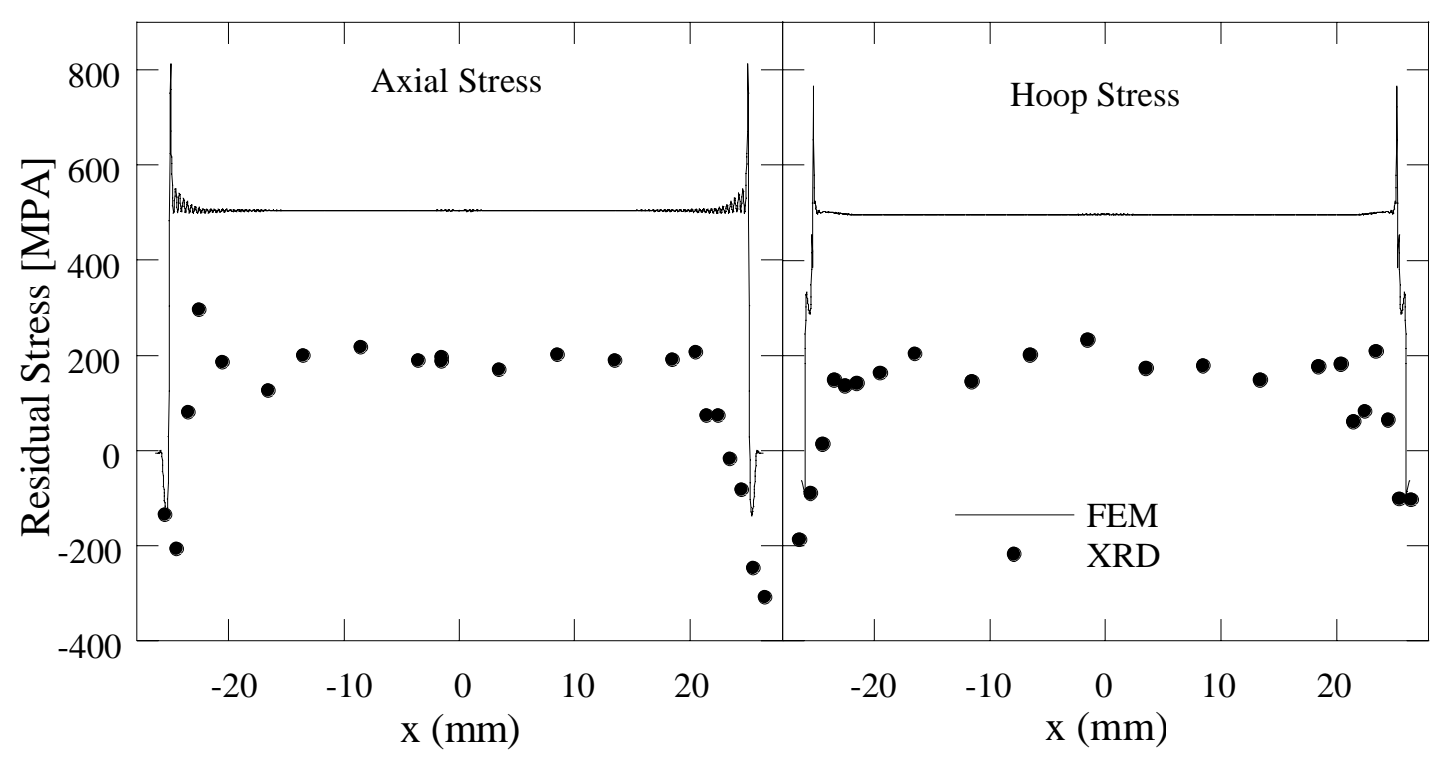

Figure 3. Calculated and measured residual stresses along clad tube outer surface

\section{Discussion}

The objective of this research was to determine and benchmark FEM predictions of residual stresses during the completion of the HIP process of an inconel 718 cladding onto a tungsten tube against X-ray diffraction stress measurements. Tensile stresses within the inconel 718 were expected because of the differences in the coefficients of thermal expansion of tungsten (Table 1) and inconel 718 (Fig. 2). The finite element predictions overestimate the measurements by more than twice the magnitude.

The finite element modeling process described within this paper uses a complex material behavior model, which includes both plasticity and creep, and which allows for the most complete simulation of the inconel 718 materials response. The simulation of the bonding process however, is simply approximated by arbitrarilly setting the time at which complete bonding occurs in the model. Although the numerical model has some flexibility to simulate both the time at which bonding occurs and crudely approximates the diffusion bond, the poor correlation between the FEM and XRD results indicates that a better representation of the diffusion bond is needed to more accurately simulate the development of residual stresses during the HIP cycle. Additional analysis in which the bond parameters were varied would are required better understand which FEM parameters must be modified to the change the final residual stresses state. However, since the goals of these analyses were to benchmark the results of the FEM analysis in order to develop a design tool for preliminary design trade-off studies, no further effort was made to more accurately similuate the bonding process or more accurately predict final stress states. Athough the analyses still do not reflect the correct physical understanding of the bonding process which may provide better correlation between predicted and measured values, the results have shown the FEM predictions to be greater than those measured. Since the values predicted by FEM analysis are larger than the measured values, the FEM analysis does provide a representative stress state that can be used to perform additional parametric or trade-off studies. The parametric studies would make sense of, or predict, trends of how the final stress state can change the tube s response to operational 
loading conditions. Finally, the FEM Analysis can be considered bounding values since it tend to over predict the residual stress states.

\section{Summary and Conclusions}

FEA was used to predict residual stress levels from hot isostatic pressing of an inconel-718-clad tungsten tube. Residual stresses in the hot pressed specimens were measured using the XRD technique to benchmark FEA calculations.

The FEM predictions of surface axial and hoop stresses in the center section of the inconel clad tube varied from 505 to $495 \mathrm{MPa}$, respectively.

The measured residual stresses in the axial and hoop direction in the same area using XRD were 190 and $160 \mathrm{MPa}$, respectively.

The FEM predictions of the axial stresses were more than twice the XRD measured stresses levels. The disparity in the comparison of predicted and measured results indicates that a better understanding of the bonding process is needed to make more accurate computations.

\section{Acknowledgment}

The authors acknowledge Ron Barber and Mike Cappiello of the Los Alamos National Laboratory for their support of this research. Harlan Horner and Bob Johnson of General Atomics were instrumental in providing samples for measurement. We would like to thank Ed Dalder of Lawrence Livermore National Laboratory for his assistance in locating difficult to find material properties at elevated temperatures. This work was supported under the auspices of the United States Department of Energy, and the Accelerator Production of Tritium Technical Project Office. The Lujan Scattering Center is a national user facility funded by the United States Department of Energy, Office of the Basic Energy Sciences - Materials Science, under contract number W-7405-ENG-36 with the University of California.

\section{References}

[1] Machine Design 1995 Materials Selector Issue Part 2, Penton Publications, 1994, p. 89.

[2] Ed Dalder, Lawrence Livermore National Laboratory, facsimile communication of LLNL-SBIP Final Report, No Author Given, Drop Hammer Formability of Inconel 718 and 718 SPF Alloys for Hughes Bros. Aircrafters, Inc., South Gate, CA 90280, 6/8/1995.

[3] W. D. Klopp, "Aerospace Structural Metals Handbook, 4, Code 4103, 1-102, 1995.

[4] Hibbitt, Karlsson \& Sorensen, Inc., ABAQUS/Standard User s Manual, 1, Version 5.8, 1998

[5] A. A. Blatherwick, A. Cers, "Fatigue, Creep and Stress-Rupture Properties of Nicrotung, Super A-286, And Inconel 718," AF Technical Report AFML-TR-65-447, Wright-Patterson Air Force Base, Ohio, 1966.

[6] "Mark's Standard Handbook for Mechanical Engineers," Ninth Edition, McGraw-Hill Book Co., p. 3-26, 1978.

[7] I.C. Noyan and J. B. Cohen, Residual Stress: Measurement by Diffraction and Interpretation , Springer-Verlag, New York, 1987. 
[8] Residual Stress Measurement by X-ray diffraction, SAE Information Report J784a, M.E. Hilley, ed., Society of Automotive Engineers, New York, August, 1971. 